\title{
RESEARCH
}

Open Access

\section{Upregulating CXCR7 accelerates endothelial progenitor cell-mediated endothelial repair by activating Akt/Keap-1/Nrf2 signaling in diabetes mellitus}

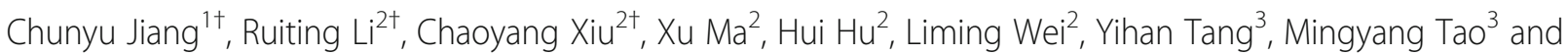
Jungong Zhao ${ }^{2^{*}}$

\begin{abstract}
Background: Endothelial progenitor cell (EPC) dysfunction contributes to vascular disease in diabetes mellitus. However, the molecular mechanism underlying EPC dysfunction and its contribution to delayed reendothelialization in diabetes mellitus remain unclear. Our study aimed to illustrate the potential molecular mechanism underlying diabetic EPC dysfunction in vivo and in vitro. Furthermore, we assessed the effect of EPC transplantation on endothelial regeneration in diabetic rats.

Methods: Late outgrowth EPCs were isolated from the bone marrow of rats for in vivo and in vitro studies. In vitro functional assays and Western blotting were conducted to reveal the association between C-X-C chemokine receptor type 7 (CXCR7) expression and diabetic EPC dysfunction. To confirm the association between cellular CXCR7 levels and EPC function, CXCR7 expression in EPCs was upregulated and downregulated via lentiviral transduction and RNA interference, respectively. Western blotting was used to reveal the potential molecular mechanism by which the Stromal-Derived Factor-1 (SDF-1)/CXCR7 axis regulates EPC function. To elucidate the role of the SDF-1/CXCR7 axis in EPC-mediated endothelial regeneration, a carotid artery injury model was established in diabetic rats. After the model was established, saline-treated, diabetic, normal, or CXCR7-primed EPCs were injected via the tail vein.

\footnotetext{
* Correspondence: zhaojungongradio@hotmail.com;

zhaojungong@sjtu.edu.cn

${ }^{\dagger}$ Chunyu Jiang, Ruiting Li and Chaoyang Xiu contributed equally to this

work.

${ }^{2}$ Department of Radiology, The Sixth People's Hospital, Affiliated to Shanghai

Jiao Tong University, 600 Yi-Shan Road, Shanghai 200233, People's Republic

of China

Full list of author information is available at the end of the article
}

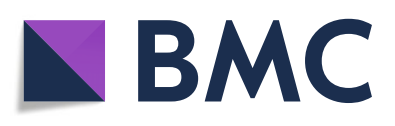

(- The Author(s). 2021 Open Access This article is licensed under a Creative Commons Attribution 4.0 International License, which permits use, sharing, adaptation, distribution and reproduction in any medium or format, as long as you give appropriate credit to the original author(s) and the source, provide a link to the Creative Commons licence, and indicate if changes were made. The images or other third party material in this article are included in the article's Creative Commons licence, unless indicated otherwise in a credit line to the material. If material is not included in the article's Creative Commons licence and your intended use is not permitted by statutory regulation or exceeds the permitted use, you will need to obtain permission directly from the copyright holder. To view a copy of this licence, visit http://creativecommons.org/licenses/by/4.0/. The Creative Commons Public Domain Dedication waiver (http://creativecommons.org/publicdomain/zero/1.0/) applies to the data made available in this article, unless otherwise stated in a credit line to the data. 
(Continued from previous page)

Results: Diabetic EPC dysfunction was associated with decreased CXCR7 expression. Furthermore, EPC dysfunction was mimicked by knockdown of CXCR7 in normal EPCs. However, upregulating CXCR7 expression reversed the dysfunction of diabetic EPCS. The SDF-1/CXCR7 axis positively regulated EPC function by activating the AKTassociated Kelch-like ECH-associated protein 1 (keap-1)/nuclear factor erythroid 2-related factor 2 (Nrf2) axis, which was reversed by blockade of AKT and Nrf2. Transplantation of CXCR7-EPCs accelerated endothelial repair and attenuated neointimal hyperplasia in diabetes mellitus more significantly than transplantation of diabetic or normal EPCs. However, the therapeutic effect of CXCR7-EPC transplantation on endothelial regeneration was reversed by knockdown of Nrf2 expression.

Conclusions: Dysfunction of diabetic EPCs is associated with decreased CXCR7 expression. Furthermore, the SDF-1/ CXCR7 axis positively regulates EPC function by activating the AKT/keap-1/Nrf2 axis. CXCR7-primed EPCs might be useful for endothelial regeneration in diabetes-associated vascular disease.

Keywords: Diabetes mellitus, Endothelial progenitor cells, CXCR7, Nrf2, Reendothelialization, Neointimal hyperplasia

\section{Background}

Diabetes mellitus (DM) is a high-incidence, chronic, metabolic disease causing many complications, such as peripheral and cardiovascular disease, that severely influence patients' quality of life [1, 2]. Interventional therapy, especially stent implantation, has been widely used for DM- associated vascular events and has significantly improved the prognosis of patients. However, unfavorable events after stent implantation, such as in-stent restenosis and late stent thrombosis, remain a serious clinical challenge $[3,4]$. A large body of evidence has shown that endothelial injury caused by DM or interventional therapy contributes to these unfavorable vascular events [5-8]. Previous studies demonstrated that endothelial progenitor cells (EPCs) are crucial in endothelial repair [6-9]. However, DM impairs EPC function and decreases circulating EPC levels in both human patients and mice with diabetes [10-12], and these effects are associated with delayed reendothelialization $[13,14]$. Thus, it is crucial to accelerate reendothelialization by understanding the potential mechanism underlying EPC dysfunction in DM.

CXC Chemokine Receptor 7 (CXCR7) is one of the most important ligand receptors for Stromal-Derived Factor-1 (SDF-1, also called CXCL12) and is expressed by EPCs [15-18]. The SDF-1/CXCR7 axis is involved in regulating the survival, adhesion, and angiogenesis of EPCs $[15,17,18]$. Moreover, dysfunctional EPCs derived from patients with hypertension have a decreased CXCR7 level, which is associated with delayed reendothelialization [19]. Dai et al. further confirmed that decreased CXCR7 expression was associated with dysfunction of late outgrowth EPCs in DM. Furthermore, elevated expression of CXCR7 enhances the resistance of EPCs to DM-induced oxidative damage and improves the therapeutic efficacy of EPCs in diabetic limb ischemia [18]. Collectively, these findings indicate that the delayed reendothelialization in DM may be associated with the impaired repair and adhesion capacities of EPCs, which are attributed to decreased CXCR7 expression. Furthermore, elevating CXCR7 expression in EPCs may accelerate reendothelialization by rescuing EPC dysfunction in DM. However, evidence supporting this hypothesis is lacking. Thus, late outgrowth EPCs were isolated to assess the association between CXCR7 expression and EPC dysfunction and determine the contribution of this mechanism to delayed reendothelialization in DM.

Few studies have reported the potential signaling mechanisms downstream of the SDF-1/CXCR7 axis involved in regulating the functional activity of EPCs. Nuclear factor erythroid 2-related factor 2 (Nrf2) is a critical redox sensor for oxidative stress [20, 21]. Activated $\mathrm{Nrf2}$, released from Kelch-like ECH-associated protein 1 (Keap-1, the repressor protein of Nrf2), translocates to the nucleus; binds to antioxidant response elements (AREs); activates the transcription of its target antioxidant genes, including heme oxygenase-1 (HO-1) and NADPH quinone oxidoreductase-1 (NQO-1) [21, 22]; and counteracts EPC dysfunction caused by reactive oxygen species (ROS) $[13,23]$. Thus, our study also assessed whether the SDF-1/CXCR7/AKT axis regulates EPC functional activity by activating the Keap-1/Nrf2 pathway.

\section{Methods and materials Study design}

A single-blind randomized study was conducted to reveal the effect of EPCs on endothelial repair and the potential role of the CXCR7/AKT/keap-1/Nrf2 axis in regulating EPC functional activity. The reendothelialization rate at day 7 and 14 and $I / M$ ratio at day 21 after EPCs transplantation were recognized as our primary outcome. Totally 125 rats were randomly divided into 
two groups: diabetic group and normal group. Then, the diabetic group was divided into six groups: control group, diabetic group, normal groups, CXCR7 group, CXCR7-EPCs ${ }^{\text {Nrf2-WT }}$ group, and CXCR7-EPCs ${ }^{\text {Nrf2-KD }}$ group. After groups and subgroups were divided, diabetic rat model was established and proved by rapid glucose meter. Then, EPCs were isolated from the bone marrow of normal or diabetic rats (10 rats each group) and used in subsequent in vitro and in vivo experiments. A carotid artery injury model was established in diabetic rats (totally, 105 rats) for subsequent in vitro study (the details are shown in supplement data 1). Finally, the results of the in vivo and in vitro studies were assessed by an analyst who was blinded to the experimental procedure.

\section{Diabetic rat model}

Ten-week-old male Sprague-Dawley (SD) rats (200-250 g) were injected intraperitoneally with $55 \mathrm{mg} / \mathrm{kg}$ streptozotocin (STZ; Sigma-Aldrich, St. Louis, USA). On days 7 and 14 , rats with fasting blood glucose levels higher than $14 \mathrm{mmol} / \mathrm{L}$ were considered diabetic and included in subsequent experiments. If fasting blood glucose levels were lower than $14 \mathrm{mmol} / \mathrm{L}$, another STZ injection was conducted to assure that diabetic rat model was constructed successfully.

\section{EPCs isolation and culture}

EPCs were isolated from the bone marrow of normal rats and rats with diabetes. The isolation, culture, and identification of EPCs were conducted as described in a previous study [23]. In brief, bone marrow was isolated from the femur and tibia and subjected to digestion, grinding, filtration, and resuspension in $10 \mathrm{~mL}$ of phosphate-buffered saline (PBS). EPCs were isolated from the cell suspension by Ficoll gradient centrifugation $(1500 \times g)$ for $10 \mathrm{~min}$ at room temperature and cultured in endothelial basal medium (Lonza Group Ltd.) containing growth factors, based on the manufacturer's instructions.

Fluorescent staining, immunocytochemistry, and flow cytometry were conducted to reveal the characteristics of the EPCs used in our study. In brief, fluorescent staining was used to detect the uptake of DiI-conjugated acetylated low-density lipoprotein (ac-LDL) (DiI-ac-LDL; Molecular Probes; Thermo Fisher Scientific, Inc.) and binding of FITC-UEA-l (Sigma-Aldrich; Merck KGaA). For immunocytochemistry, cells were fixed, incubated with primary antibodies overnight, and then incubated with secondary antibodies for $1 \mathrm{~h}$. The cells were then washed three times and visualized using a fluorescence microscope (Leica AF6000). Antibodies specific for CD31 (1:100, Cat No. ab222783), CD34 (1:100, Cat No. ab81289), and vWF (1:100, Cat No. ab216566) were obtained from Abcam (Cambridge, Cambs., UK). For flow cytometry, cells were fixed and were then incubated with the following primary antibodies: BB515-conjugated mouse anti-human CD31 (Cat No. 565408), APCconjugated mouse anti-human CD34 (Cat No. 560940), PE-conjugated mouse anti-human VEGFR-2 (Cat No. 560872), and FITC-conjugated mouse anti-human CD45 (Cat No. 554883). The antibodies were obtained from BD Biosciences (San Jose, CA, USA). Nonspecific fluorescence was assessed by incubation of similar cell aliquots with isotype-matched mouse monoclonal antibodies. Cells were washed with PBS and analyzed by using a GuavaeasyCyte ${ }^{\mathrm{mm}}$ Flow Cytometer (Millipore, Billerica, MA, USA).

\section{Lentiviral transduction and RNA interference}

Lentiviral transduction and RNA interference were conducted to upregulate or downregulate, respectively, the expression of CXCR7 to confirm the association between CXCR7 expression and EPC dysfunction.

Recombinant lentivirus encoding CXCR7 was constructed using the pLVX-EGFP-3FLAG-Puro vector (Shanghai Sunbio Medical Biotechnology, Shanghai, China). In brief, EPCs were seeded into 24-well plates at a density of $1 \times 10^{5}$ cells/well and incubated overnight. EPCs were then transduced overnight with purified lentiviral vectors that expressed recombinant CXCR7 at a multiplicity of infection of 25 in the presence of $3 \mu \mathrm{g} / \mathrm{mL}$ polybrene (Sigma, MO, USA). After $24 \mathrm{~h}$ of infection, the medium was replaced with $2 \mathrm{~mL}$ of fresh medium. The lentiviral transduction efficiency was determined by assessing lentiviral expression of green fluorescent protein (GFP). Apparent GFP expression was observed $48 \mathrm{~h}$ after transduction and peaked $72 \mathrm{~h}$ after transduction. The levels of CXCR7 expression were confirmed by Western blot analysis.

RNA interference was conducted to downregulate the expression of CXCR7 in EPCs. The sequences of the RNA interference and negative control (NC) constructs were as follows: CXCR7 siRNA sense, 5'-GGAAGA UCAUCUUCUCCUATT-3' and antisense, 5'-UAGGAGAAGAUGAUCUUCCGG-3'; NC, 5'-UUCUCCGAAC GUGUCACGUTT-3'; antisense, 5' ${ }^{\prime}$-ACGUGACACG UUCGGAGAATT-3'. Transfection was performed as described previously [24]. In brief, siRNA transfection was performed using Lipofectamine 2000 reagent (Invitrogen, Carlsbad, CA, USA) following the manufacturer's protocols. EPCs were plated into 6-well plates at a density of $5 \times 10^{5}$ cells/well and incubated overnight. The diluted siRNA constructs and Lipofectamine 2000 reagent were mixed at a ratio of $1: 1(4 \mathrm{pmol}$ siRNA to $4 \mu \mathrm{L}$ of $\mathrm{Li}$ pofectamine 2000) and incubated for $20 \mathrm{~min}$ at room temperature. Finally, $400 \mu \mathrm{L}$ of the mixture was added to each well to achieve a final volume of $2 \mathrm{~mL}$, and the cells 
were continuously incubated for $48 \mathrm{~h}$ before starting subsequent experiments.

Nrf2 expression in CXCR7-primed EPCs was knocked down to reveal the detailed mechanism underlying the regulation of EPC functional activity by the SDF-1/ CXCR7 axis. In brief, EPCs were infected with lentiviruses containing shRNA against Nrf2 or nonsense shRNA (Genomeditech, Shanghai, China). The sequences of the Nrf2 and nonsense shRNA constructs are as follows:

Nrf2-shRNA 1, 5'- GGGTAAGTCGAGAAGTGT TTG -3'

Nrf2-shRNA 2, 5' - GGACCTAAAGCACAGCCAACA $-3^{\prime}$

Nrf2-shRNA 3, 5' - GCAAGAAGCCAGATACAAAGA $-3^{\prime}$

Nonsense shRNA, 5' - TTCTCCGAACGTGTCACG TAG - 3 '

Transfection was performed following a previously described protocol. After transfection for $48 \mathrm{~h}$, the expression of Nrf2 was determined by Western blot analysis. Then, EPCs were infected with lentiviruses containing the shRNA against Nrf2 with the highest knockdown efficiency (lowest expression of Nrf2) or with nonsense shRNA determined by western blot analysis.

\section{EPC adhesion assay}

The adhesion of EPCs to human umbilical vein endothelial cells (HUVECs, Cell Resource Center of Shanghai Institute for Biological Sciences, Chinese Academy of Sciences; Cat No. 3131C0001000200023) was assessed by plating these cells in 24-well plates. First, HUVECs were plated in 24-well plates to form a monolayer. Then, the nonattached cells were washed away with PBS. DAPI $(10 \mu \mathrm{g} / \mathrm{mL})$ was used for staining HUVECs. EPCs were cultured in medium containing DiI dye $(4 \mathrm{mg} / \mathrm{mL})$ for $30 \mathrm{~min}$ at $37^{\circ} \mathrm{C}$ following the manufacturer's protocol. DiI-labeled EPCs were digested and harvested after three washes with PBS. DiI-labeled EPCs were then added to the plate and were then incubated with the HUVEC monolayer for $2 \mathrm{~h}$, after which nonattached EPCs were washed away with PBS. Adherent EPCs were counted in five random fields under an Olympus microscope at $x$ 400 magnification.

\section{Repair capacity of EPCs in vitro}

The repair capacity of EPCs in vitro was assessed using a scratch assay. In brief, EPCs were plated in 96-well plates to form a monolayer after $48 \mathrm{~h}$ of culture. Then, the confluent monolayer was scratched using a p200 pipette tip $(1 \mathrm{~mL})$ containing serum-free medium. The wound area was imaged under a microscope (magnification, $\times 100$; Olympus Corporation) at baseline $(0 \mathrm{~h})$ and after $24 \mathrm{~h}$, and the data were then analyzed using ImageJ software (National Institutes of Health).

\section{Western blot analysis}

EPCs were incubated for $24 \mathrm{~h}$ before protein extraction with a protein extraction kit (Solarbio, Beijing, China) and quantification with a bicinchoninic acid protein assay kit (Solarbio). Protein extracts were subjected to SDS-PAGE (KeyGEN, Nanjing, China) followed by transfer onto polyvinylidene fluoride membranes (Roche, IN, USA). The following primary antibodies (all obtained from Abcam (Life Technologies, CA, USA)) were used: anti-CXCR7 (1:1500, Cat No. ab138509), anti-GAPDH (1:10000, Cat No. ab8245), anti-p-Akt (1:5000, Cat No. ab38449), anti-Keap-1 (1:1000, Cat No. ab139729), antiNrf2 (1:1000, Cat No. ab92946), anti-HO-1 (1:2000, Cat No. ab189491), and anti-NQO-1 (1:1000, Cat No. ab80588). Subsequently, the membranes were incubated with secondary antibodies (1:1000, Cat No. bs-0346RHRP; Beijing Boaosen Biotechnology Co., Ltd.) for $2 \mathrm{~h}$ at room temperature. Protein bands were visualized using an Epson Photo 1650 scanner (Seiko Epson Corp., Japan).

\section{Rat model and treatment regimens}

The vascular injury rat model was used to assess the effect of putative EPCs on intimal repair. After establishment of the rat model of type I diabetes described above, the vascular injury model was established as described previously [23]. In brief, the vascular endothelium was injured by a guidewire as used in percutaneous transluminal angioplasty. The operation was performed under anesthesia with pentobarbital sodium. Then, the bifurcation of the left carotid artery was exposed, and the common, internal, and external carotid arteries were separated to temporarily restrict blood flow. The common carotid artery was denuded by three repeated passages of the $0.38-\mathrm{mm}$ flexible angioplasty guidewire through the external carotid artery. The denuded segment encompassed a total length of $5 \mathrm{~mm}$ from the carotid bifurcation. The external carotid artery was permanently ligated after the guidewire was removed, and the temporary ligatures were released to allow restoration of blood flow followed by skin suture.

Then, $1.5 \mathrm{~mL}$ of saline (control group) or a cell suspension $\left(1 \times 10^{6}\right.$ cells $\left./ \mathrm{mL}\right)$ of normal EPCs (derived from normal rats; normal group), diabetic EPCs (derived from rats with diabetes; diabetic group), CXCR7-EPCs (derived from rats with diabetes, CXCR7-EPC group), CXCR7-EPCs ${ }^{\mathrm{Nr} f 2-W T}$ (derived from normal rats; CXCR7-EPC ${ }^{\text {Nrf2-WT }}$ group), or CXCR7-EPCs ${ }^{\text {Nrf2-KD }}$ (derived from normal rats; CXCR7-EPC ${ }^{\mathrm{Nrf2}-\mathrm{KD}}$ group) was injected into the circulation via the tail vein immediately 
and $12 \mathrm{~h}$ after establishment of the vascular injury model.

\section{Assessment of reendothelialization and neointimal hyperplasia}

The reendothelialization rate was assessed by Evans blue staining on days 7 and 14 after treatment. In brief, 0.5 $\mathrm{mL}$ of $0.5 \%$ Evans blue dye was injected intravenously via the tail vein $30 \mathrm{~min}$ before sacrifice. Cardiac perfusion was then performed via the bilateral jugular vein with formaldehyde fixation for $5 \mathrm{~min}$ followed by washing with PBS until the effluent ran clear. The common carotid artery was harvested $4 \mathrm{~mm}$ from the bifurcation after measurement by a Vernier caliper and opened longitudinally using microscissors. Then, an image of the vessel was acquired with a stereomicroscope (DVM6, Leica). Digital images were analyzed using NIH ImageJ 1.63 software. The total endothelial area in all groups was first measured and analyzed. After confirming that the total endothelial area did not differ significantly among the groups, the Evans blue-stained and unstained areas were measured to calculate the reendothelialization rate (unstained area/total area).

Neointimal hyperplasia was assessed using hematoxylin and eosin (HE) staining and Masson trichrome staining on day 21 after treatment. Denuded arteries were harvested from rats and immersed in formalin for $24 \mathrm{~h}$. The neointimal thickness was assessed using the intimal area-to-medial area ratio $(I / M)$ in HEstained axial sections. A pathologist blinded to the treatment regimen assessed all specimens using NIH ImageJ 1.63 software.

\section{Statistical analysis}

Data are expressed as the mean \pm standard deviation values. One-way or multi-way analysis of variance (ANOVA) with post hoc LSD comparisons with polynomial contrasts was used to determine significant differences between pairs of subgroups at each time point. $p$ values $<0.05$ were considered to be statistically significant. SPSS 20.0 software (IBM Corp., Armonk, NY, USA) was used to perform statistical analysis.

\section{Results}

\section{Characteristics of EPCs}

EPC clusters appeared 21 days after culture initiation (Fig. 1a). Three markers (v-WF, CD34, and CD31) were used for immunofluorescence detection to validate the harvested EPCs (Fig. 1b). Flow cytometric analysis showed that the EPCs were positive for CD34, VEGFR2, and CD133 but negative for CD45 (Fig. 1c). In addition, these cells showed positive staining for ac-LDL and the endothelial-specific lectin UEA-1 (Fig. 1d), further confirming their identity as late outgrowth EPCs (LOCs).
DM downregulated CXCR7 expression and attenuated the functional activity of EPCs

Late outgrowth EPCs were isolated from the bone marrow of normal rats and rats with diabetes to determine the effect of DM on CXCR7 expression and the functional activity of EPCs. The expression of CXCR7 was obviously decreased in EPCs derived from rats with diabetes compared with EPCs from normal rats (Fig. 2a, b). In addition, the adhesion capacity of diabetic EPCs was attenuated compared with that of normal EPCs (normal group vs diabetic group: $23.8 \pm 4.9$ vs $9.4 \pm 1.2$ cells/field, $p=0.014$ ) (Fig. 2 c, d). Furthermore, the scratch assay showed that the in vitro repair capacity of EPCs was attenuated in the context of DM (normal group vs diabetic group, $52.83 \pm 7.81 \%$ vs $24.93 \pm 3.69 \%, p=0.038$; Fig. 2 e, f).

To confirm the association between CXCR7 expression and EPC dysfunction, gain- and loss-of-function studies were performed by siRNA-mediated knockdown in EPCs obtained from normal rats and lentivirusmediated overexpression in EPCs obtained from rats with diabetes. The studies showed that EPC dysfunction was mimicked by knockdown of CXCR7. Downregulating CXCR7 expression impaired the adhesion (normal group vs SiCXCR7 group, $16.8 \pm 3.9$ vs $4.6 \pm 1.1$ cells/ field, $p=0.017$ ) and repair (normal group vs SiCXCR7 group, $52.83 \pm 7.81 \%$ vs $35.31 \pm 4.27 \%, p=0.041$ ) capacities of normal EPCs. In contrast, increasing CXCR7 expression significantly reduced the dysfunction of diabetic EPCs, including restoring the adhesion (diabetic group vs Lv-CXCR7 group, $3.9 \pm 1.2$ vs $17.1 \pm 3.2$ cells/field, $p=0.01$ ) and repair (diabetic group vs Lv-CXCR7 group, $24.93 \pm 3.69 \%$ vs $67.5 \pm 8.49 \%, \quad p=0.026) \quad$ capacities in vitro (Fig. $2 \mathrm{c}-\mathrm{f}$ ). Collectively, these results indicated that the dysfunction of diabetic EPCs was attributed to the decrease in CXCR7 expression.

\section{Upregulating CXCR7 accelerated endothelial repair by EPCs and attenuated neointimal hyperplasia in DM}

Saline-treated EPCs (control group), normal EPCs (normal group), diabetic EPCs (diabetic group), and CXCR7primed EPCs (CXCR7 group) were transplanted into rats with diabetes after induction of vascular injury to determine whether the elevated CXCR7 level accelerated EPC-mediated endothelial repair. The reendothelialization rate, $I / M$ ratio, and neointimal area were used to assess endothelial regeneration and neointimal formation in the different groups. CXCR7-EPC transplantation effectively accelerated reendothelialization on day 7 (control group vs CXCR7 group, $66.41 \pm 7.38 \%$ vs $94.66 \pm 9.6 \%, p=0.0298$ ) but not on day 14 (control group vs CXCR7 group, $86.46 \pm 10.35 \%$ vs $98.56 \pm$ $10.67 \%, p=0.398)$ after vascular injury in the DM model (Fig. $3 \mathrm{a}-\mathrm{c})$. Furthermore, CXCR7-EPC transplantation 


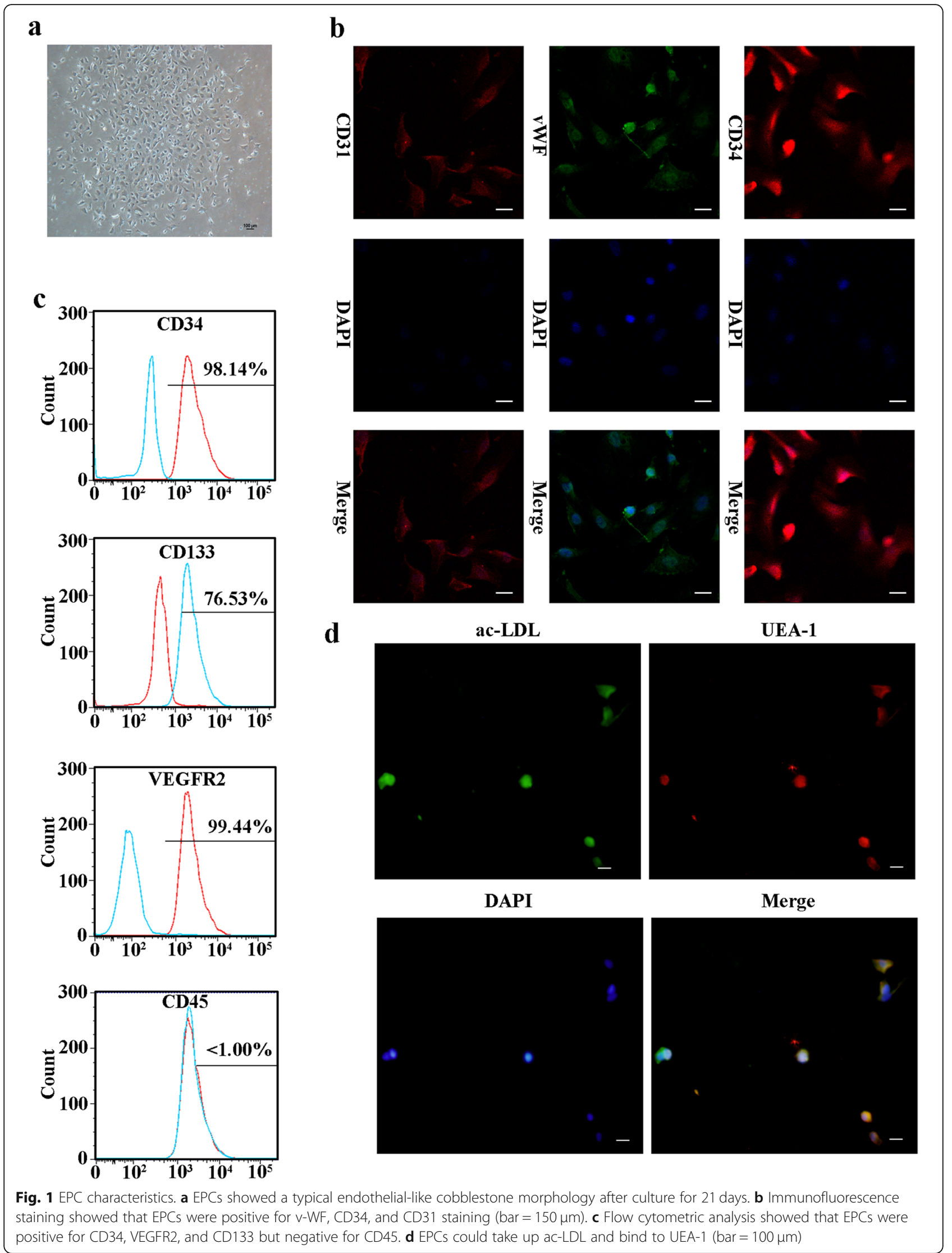




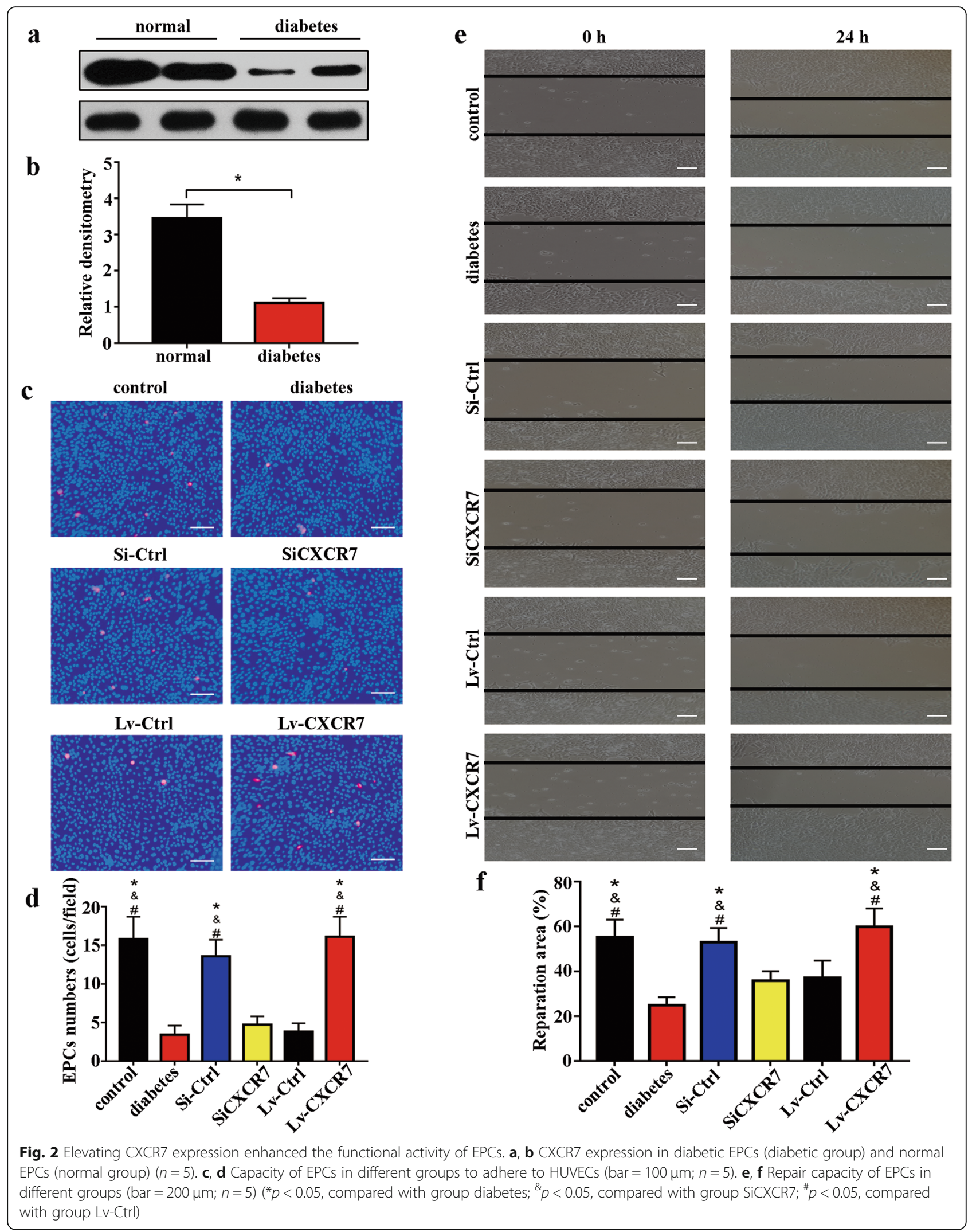




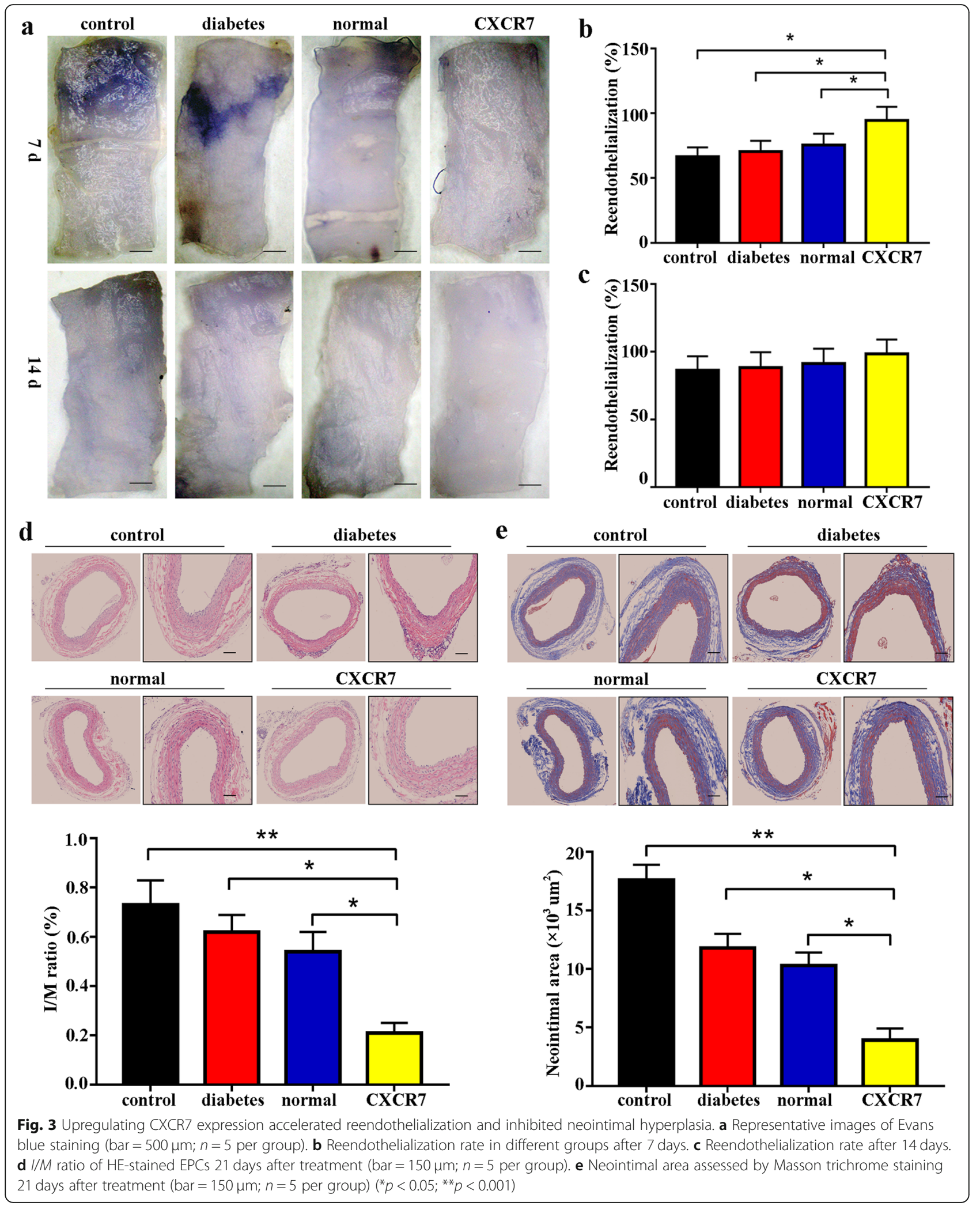

significantly reduced neointimal formation at 21 days after treatment (control group vs CXCR7 group: $I / M$ ratio, $\quad 75.63 \pm 10.45 \% \quad$ vs $23.85 \pm 5.67 \%, \quad p<0.001$; neointimal area, $17.75 \pm 2.19$ vs $4.72 \pm 1.33, p<0.001$ ). However, transplantation of normal or diabetic EPCs failed to accelerate endothelial repair and attenuate 
neointimal hyperplasia in the DM model (Fig. 3d, e). Collectively, these findings indicated that upregulating CXCR7 expression reversed diabetic EPC dysfunction and accelerated EPC-mediated endothelial repair in DM.

\section{Elevation of CXCR7 stimulated HO-1 and NQO-1 expression by activating the Akt-associated Keap-1/Nrf2 axis}

The underlying mechanism by which the SDF-1/CXCR7 axis regulates EPC function remains largely unknown. Previous studies showed that the Keap-1/Nrf2 axis was crucial in positively regulating EPC function by activating target antioxidant genes. Thus, the SDF-1/CXCR7 axis was hypothesized to positively regulate EPC function by activating the Akt-associated Keap-1/Nrf2 axis. To confirm this hypothesis, EPCs were obtained from the bone marrow of wild-type rats, and the protein levels of relevant signaling molecules, including p-Akt, Keap-1, Nrf2, HO-1, and NQO-1, were assessed by Western blot analysis.

The p-Akt level in CXCR7-EPCs was higher than that in normal EPCs after pretreatment with SDF-1. Moreover, pretreatment with SDF-1 significantly decreased the Keap-1 level but promoted the accumulation of nuclear Nrf-2 in CXCR7-EPCs compared with normal EPCs. The HO-1 and NOQ-1 levels were also higher in CXCR7-EPCs than in normal EPCs (Fig. 4a). Then, Akt was blocked with LY29400 in normal EPCs and CXCR7EPCs. Blockade of Akt inactivated the Keap-1/Nrf2 axis and decreased the expression of its downstream target genes in both normal and CXCR7-EPCs (Fig. 4a). Collectively, these results indicated that upregulation of CXCR7 controlled EPC functional activity by activating the Akt-associated Keap-1/Nrf2 axis.

\section{Knockdown of Nrf2 attenuated the functional activity and endothelial repair capacity of CXCR7-EPCs}

Nrf2 expression was knocked down with shRNA to further confirm the association between the Keap-1/Nrf2 axis and the SDF-1/CXCR7 pathway. Knockdown of Nrf2 expression significantly decreased the Nrf2 level, resulting in reductions in HO-1 and NOQ-1 levels in both normal EPCs and CXCR7-EPCs (Fig. 4b). Moreover, knockdown of Nrf2 abolished the protective effects of CXCR7 on EPC functional activity in vitro (Fig. 5). Both the adhesion capacity (CXCR7-EPC ${ }^{\text {Nrf2-KD }}$ group vs CXCR7-EPC ${ }^{\mathrm{Nr} 2-W \mathrm{~W}}$ group, $15.94 \pm 2.78$ vs $34.5 \pm 3.28$ cells/field, $p=0.036$ ) and the repair capacity (CXCR7EPC $^{\text {Nrf2-KD }}$ group vs CXCR7-EPC ${ }^{\text {Nrf2-WT }}$ group, $19.17 \pm$ $4.69 \%$ vs $37.54 \pm 6.42 \%, p=0.029$ ) were significantly attenuated in CXCR7-EPCs after blockade of Nrf2 (Fig. 5).

Saline-treated EPCs (control group), CXCR7$\mathrm{EPCs}^{\text {Nrf2-WT }}$ (CXCR7-EPC ${ }^{\mathrm{Nrf2}-\mathrm{WT}}$ group), or CXCR7-
EPCs $^{\text {Nrf2-KD }}$ (CXCR7-EPC ${ }^{\text {Nrf2-KD }}$ group) were transplanted into the rats with both diabetes and carotid artery injury to determine whether knockdown of Nrf2 impairs CXCR7-EPC-mediated endothelial repair in DM. CXCR7-EPC ${ }^{\mathrm{Nrf2}-\mathrm{WT}}$ transplantation accelerated reendothelialization (CXCR7-EPC ${ }^{\text {Nrf2-WT }}$ vs control group: 7 days, $91.42 \pm 10.03 \%$ vs $52.19 \pm 4.55 \%, p<0.001 ; 14$ days, $97.05 \pm 11.86 \%$ vs $89.7 \pm 8.16 \%, p=0.429)$ and inhibited neointimal hyperplasia (CXCR7-EPC ${ }^{\mathrm{Nrf2}-\mathrm{WT}}$ vs control group: $I / M$ ratio, $23.85 \pm 5.67 \%$ vs $75.63 \pm 10.45 \%, p<$ 0.001 ; neointimal area, $4.72 \pm 1.33$ vs $17.75 \pm 2.19 \times$ $\left.10^{3} \mu^{2}, p<0.001\right)$ compared with the corresponding parameters in the control group. However, blockade of Nrf2 significantly impaired EPC-mediated endothelial repair. The reendothelialization rate in the CXCR7EPC $^{\mathrm{Nr} 22-\mathrm{KD}}$ group was lower than that in the CXCR7$\mathrm{EPC}^{\mathrm{Nr} 2-W \mathrm{WT}}$ group 7 days after treatment (CXCR7EPC $^{\text {Nrf2-WT }}$ vs CXCR7-EPC ${ }^{\text {Nrf2-KD }} 7$ days, $91.42 \pm 10.03 \%$ vs $61.88 \pm 10.15 \%, p<0.001$; 14 days, $97.05 \pm 11.86 \%$ vs $91.03 \pm 10.52 \%, p=0.53)$. However, the reendothelialization rate was not significantly different between the CXCR7-EPCs ${ }^{\text {Nrf2-KD }}$ and control groups (CXCR7$\mathrm{EPC}^{\mathrm{Nr} 2-\mathrm{KD}}$ vs control group: 7 days, $61.88 \pm 10.15 \%$ vs $52.19 \pm 4.55 \%, p=0.426 ; 14$ days, $91.03 \pm 10.52 \%$ vs $89.7 \pm$ $8.16 \%, p=0.658$ ) (Fig. $6 a-c)$. In contrast, the $I / M$ ratio in the CXCR7-EPC ${ }^{\text {Nrf2-KD }}$ group was higher than that in the CXCR7-EPC ${ }^{\text {Nrf2-WT }}$ group on day 21 (CXCR7-EPC ${ }^{\text {Nrf2-WT }}$ vs CXCR7-EPC ${ }^{\mathrm{Nrf2}-\mathrm{KD}}, 23.85 \pm 5.67 \%$ vs $62.92 \pm 4.72 \%$, $p<0.001$ ), and the neointimal volume in the CXCR7$E^{\text {Nrf2-KD }}$ group was also higher than that in the CXCR7-EPC ${ }^{\text {Nrf2-WT }}$ group on day 21 (CXCR7-EPC ${ }^{\text {Nrf2-WT }}$ vs CXCR7-EPC ${ }^{\mathrm{Nr} 2-\mathrm{KD}}, \quad 4.72 \pm 1.33$ vs $15.86 \pm 2.12 \times$ $10^{3} \mu \mathrm{m}^{2}, p<0.001$ ) (Fig. 6d, f). Collectively, these findings confirmed that the Keap-1/Nrf2 axis is downstream of the SDF-1/CXCR7 pathway and is involved in regulating EPC function in vivo and in vitro.

\section{Discussion}

Recent developments in the characterization of human peripheral blood mononuclear cell-derived EPCs have led to the identification of two types of EPCs: early EPCs and late outgrowth EPCs [25-28]. The surface antigen molecules most commonly used to immunophenotype EPCs include CD34, CD133, KDR, CD31, CD45, and CD14 [29, 30]. Most previous studies considered early EPCs to be positive for CD14 and CD45 $[28,30]$ and late outgrowth EPCs to be positive for CD34, CD133, and KDR but negative for CD45 [29, 31]. All of these different EPC subtypes contribute to endothelial repair of vascular injury; late outgrowth EPCs can differentiate into endothelial cells, but early EPCs exert beneficial effects through their proangiogenic paracrine activity [32, 33]. Here, we assessed the association between CXCR7 expression and the repair capacity of late outgrowth EPCs 


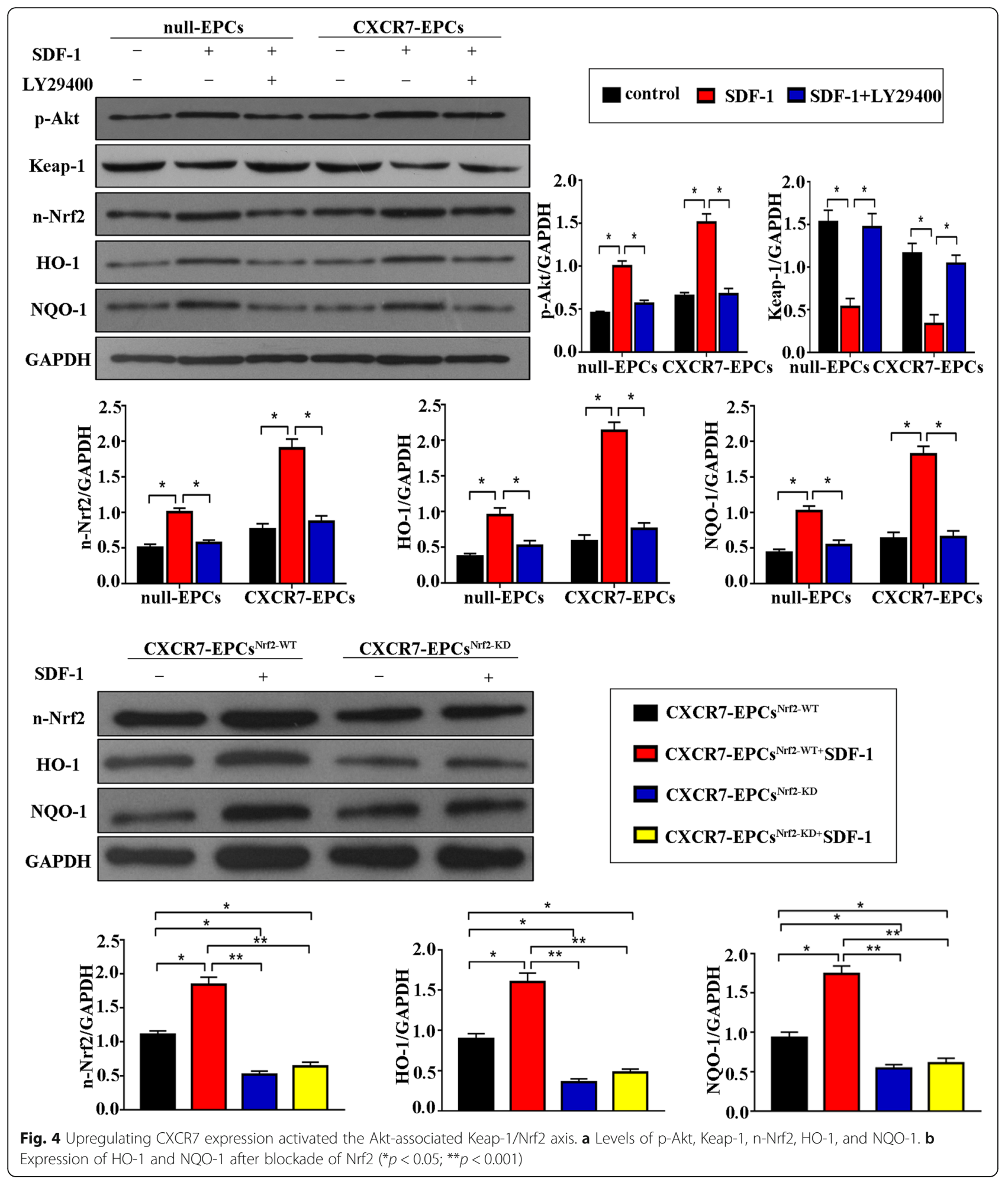

in DM. Furthermore, we explored the potential signaling molecules downstream of the SDF-1/CXCR7 axis that are involved in regulating EPC functional activity. We found that DM impaired the repair capacity of EPCs both in vivo and in vitro, and this impairment was attributed to decreased CXCR7 expression. Furthermore, our results confirmed the critical role of the AKT/Keap$1 / \mathrm{Nrf2}$ axis as the downstream signaling target of SDF1/CXCR7 in regulating EPC functional activity. Most importantly, we found that transplantation of CXCR7- 


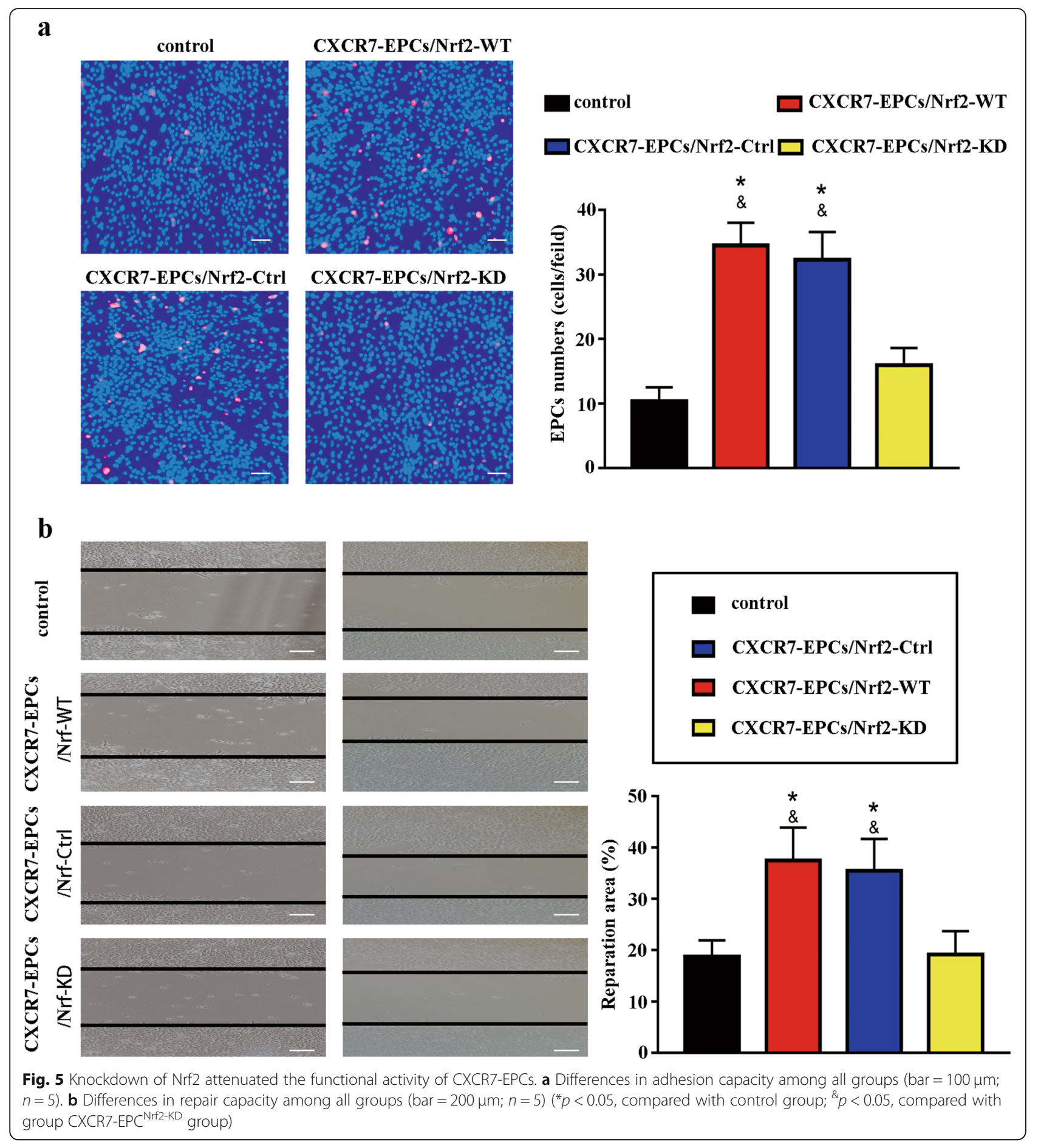

primed EPCs but not normal or diabetic EPCs was an effective method to promote reendothelialization and inhibit neointimal hyperplasia in DM.

Many studies have shown that DM impairs EPC functions in humans and mice [34, 35]. Specific important molecules, such as CXCR4, NO, and p66Shc, have been identified to control the dysfunction of cultured and circulating putative EPCs [11]. Several recent studies have indicated that the SDF-1/CXCR7 axis is involved in regulating EPC functions, including proliferation, adhesion, angiogenesis, and endothelial repair, both in vitro and in vivo [17-19]. For example, Dai et al. pointed out that DM attenuates CXCR7 expression and impairs the angiogenic function of EPCs [18]. However, the role of the SDF-1/CXCR7 axis in regulating EPC-mediated endothelial repair is still unclear. Our results confirmed 


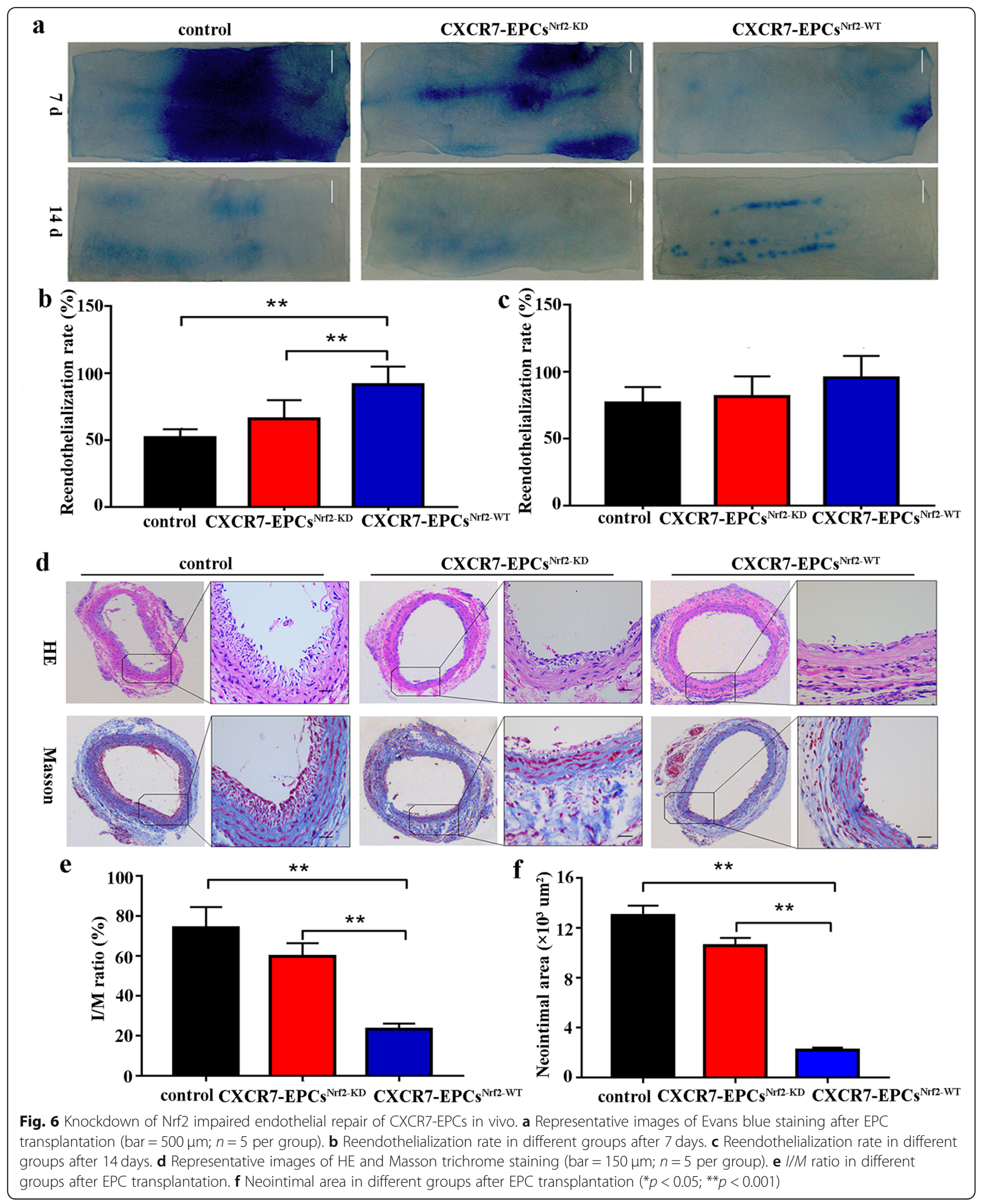

that decreasing CXCR7 expression in EPCs contributes to delayed reendothelialization in DM. Furthermore, our in vitro study revealed that the delayed endothelial repair in DM is attributed mainly to the impaired adhesion and repair capacities of EPCs, because elevating CXCR7 expression in EPCs restored their repair capacity both 
in vitro and in vivo. However, the detailed molecular mechanism by which CXCR7 expression is downregulated in diabetic EPCs remains unclear and needs further exploration.

Heterodimerization of CXCR7 with CXCR4 induces the internalization and degradation of CXCR4 [36], thus interfering with CXCR4-induced Gai protein-mediated signaling [37, 38]. However, heterodimerization of CXCR7 with CXCR4 favors $\beta$-arrestin recruitment [39], stimulating Akt phosphorylation [40] and integrin activation [41]. On the other hand, the CXCL12 scavenging function of CXCR7 reinforces CXCR4-mediated signaling by preventing downregulation of CXCR4 surface expression and function mediated through excessive CXCL12 concentrations [42]. Though the crosstalk between the SDF-1/CXCR4 and SDF-1/CXCR7 axes in regulating EPC function has been well illustrated by previous studies, the downstream mechanism by which the SDF-1/CXCR7 axis is involved in regulating EPC functional activity remains largely unknown. Wang et al. pointed out that Nrf2 protects EPCs against cellular dysfunction by decreasing cellular senescence under hyperglycemic conditions [22]. Moreover, activated Nrf2 can bind to AREs and increase HO-1 and NQO-1 expression, thus exerting a cytoprotective effect via the production of bioactive products or depletion of intracellular sulfhydryl pools [20,21]. Dai et al. pointed out that elevating CXCR7 expression improves EPC functional activity, including their angiogenic and migratory activity, by stimulating HO-1 and NQO-1 expression [18]. Our results also confirm that the SDF-1/CXCR7 axis regulates EPC functional activity by activating the Keap-1/ Nrf2 pathway, because upregulation of CXCR7 stimulated Akt phosphorylation, Keap-1 inactivation, Nrf2 accumulation, and HO-1 and NQO-1 expression, accompanied by enhancement of EPC functional activity, which was reversed by blocking Akt or Nrf2 with LY2900 or shRNA, respectively. Different from the results of Dai et al.'s study, our results confirmed that the SDF-1/CXCR7 axis positively modulates the adhesion and repair capacities but not the angiogenic ability of EPCs by activating Keap-1/Nrf2-associated antioxidant genes, including HO-1 and NQO- 1 . However, the molecular mechanism by which $\mathrm{HO}-1$ and NQO-1 enhance the adhesion and repair capacities of EPCs remains unclear and needs further study.

Hyperglycemia is an important risk factor for vascular disease and contributes to the development of atherosclerosis and vascular remodeling after vascular injury. Transplantation of exogenous EPCs was evaluated as a potential method to promote endothelial repair by directly elevating the circulating EPC level in a rabbit model and a hypercholesterolemic rat model [43, 44]. However, the role of EPC transplantation in regulating endothelial repair under hyperglycemic conditions remains unclear. In this study, we initially applied transplanted EPCs transfected with the CXCR7 gene to treat vascular injury under hyperglycemic conditions. Unfortunately, our results showed that neither normal EPCs nor diabetic EPCs accelerated endothelial repair in the diabetic rat model. However, the therapeutic effect of CXCR7-primed EPC transplantation on reendothelialization in DM was promising. These results may be partially due to DM-induced impairment of the adhesion and regeneration capacities of EPCs but not of CXCR7primed EPCs, which was well illustrated in our in vitro

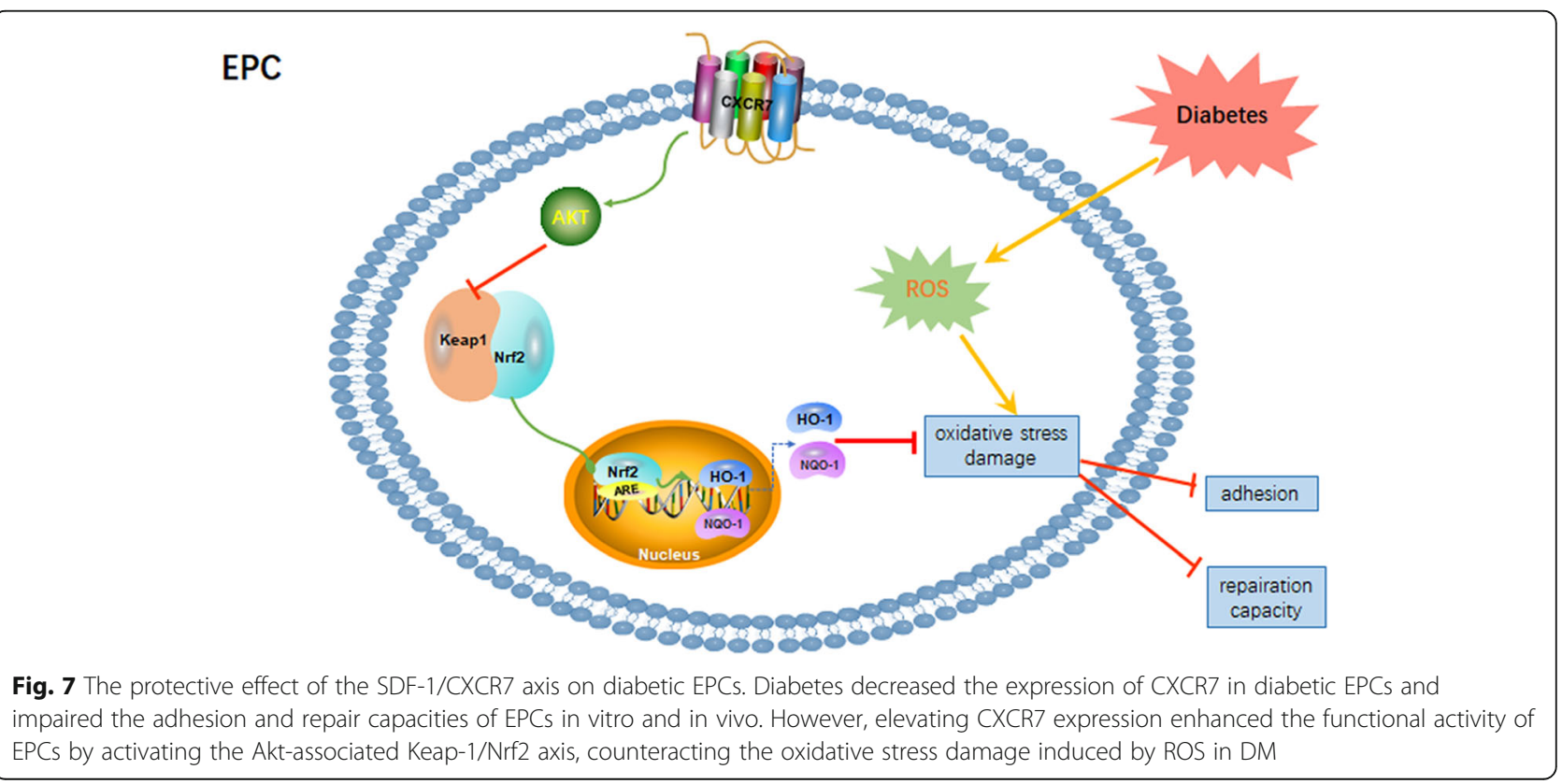


study. These results are also well supported by the findings of Dai et al., who showed that upregulating CXCR7 expression abolished the increase in the ROS level in CXCR7-EPCs exposed to ox-LDL or high glucose (HG) by stimulating HO-1 and NQO-1 expression [18]. Collectively, these data suggest that CXCR7 improves the efficiency of EPC transplantation for the treatment of diabetes-associated vascular complications, such as peripheral artery disease, coronary artery disease, vascular restenosis, and thrombosis, after interventional therapy.

\section{Conclusions}

As shown in Fig. 7, elevating CXCR7 expression preserved the functional activity of diabetic EPCs and protected against diabetes-associated oxidative stress damage by activating the Keap-1/Nrf2 axis in vivo and in vitro. Furthermore, transplantation of CXCR7-EPCs was an effective method to promote reendothelialization, indicating the potential future use of CXCR7-EPCs to treat diabetes-associated vascular disease.

\section{Abbreviations}

EPCS: Endothelial progenitor cells; LOCs: Late outgrowth EPCs; CXCR7: C-X-C chemokine receptor type 7; SDF-1: Stromal-Derived Factor-1; Keap-1: Kelchlike ECH-associated protein 1; Nrf2: Nuclear factor erythroid 2-related factor 2; DM: Diabetes mellitus; ARE: Antioxidant response element; HO-1: Heme oxygenase - 1; NQO-1: NADPH quinone oxidoreductase 1; ROS: Reactive oxygen species; GFP: Green fluorescent protein; HUVEC: Human umbilical vein endothelial cell

\section{Supplementary Information}

Supplementary information accompanies this paper at https://doi.org/10. 1186/s13287-021-02324-7.

Additional file 1.

Additional file 2.

Additional file 3.

\section{Acknowledgements}

Not applicable.

\section{Authors' contributions}

$C J, X M, C X, R L, Y T$, and MT performed the experiments. JZ, CX, and CJ designed the experiments. RL, HH, LW, YT, and MT conducted the statistical analysis of the data. CJ, XM, CX, RL, YT, and MT drafted the manuscript. JZ, $\mathrm{CJ}, \mathrm{RL}$, and CX conceived the study, participated in its design and coordination, and helped to draft the manuscript. All authors read and approved the final manuscript.

\section{Funding}

This study was supported by the National Natural Science Foundation of China (grant No. 81271683 and 81671791) and the Shanghai Key Discipline of Medical Imaging (grant No. 2017ZZ02005).

\section{Availability of data and materials}

The datasets used and analyzed in current study are available from the corresponding author based on reasonable request.

\section{Declarations}

\section{Ethics approval and consent to participate}

The procedures involving animals were executed following the ethical standards of Guide for the Care and Use of Laboratory Animals and approved by the Shanghai Sixth Peoples Hospital (No. 2016-0012).

\section{Consent for publication}

Not applicable.

\section{Competing interests}

The authors declare that they have no competing interests.

\section{Author details}

'Department of Interventional Therapy, Shanghai Ninth People's Hospital, Shanghai Jiao Tong University of Medicine, No. 639 Zhi Zao Ju Road, Shanghai 200233, People's Republic of China. ${ }^{2}$ Department of Radiology, The Sixth People's Hospital, Affiliated to Shanghai Jiao Tong University, 600 Yi-Shan Road, Shanghai 200233, People's Republic of China. ${ }^{3}$ Institute of Translational Medicine, Medical College, Yangzhou University, Yangzhou 225001, People's Republic of China.

Received: 1 September 2020 Accepted: 2 April 2021

Published online: 03 May 2021

References

1. Yang SL, Zhu LY, Han R, Sun LL, Li JX, Dou JT. Pathophysiology of peripheral arterial disease in diabetes mellitus. J Diab. 2017;9(2):133-40. https://doi. org/10.1111/1753-0407.12474.

2. Glovaci D, Fan W, Wong ND. Epidemiology of diabetes mellitus and cardiovascular disease. Curr Cardiol Rep. 2019;21 (4):21. https://doi.org/10.1 007/s11886-019-1107-y.

3. Byrne RA, Joner M, Kastrati A. Stent thrombosis and restenosis: what have we learned and where are we going? The Andreas Gruntzig Lecture ESC 2014. Eur Heart J. 2015;36(47):3320-31. https://doi.org/10.1093/eurheartj/ ehv511.

4. Jukema JW, Ahmed TA, Verschuren JJ, Quax PH. Restenosis after PCI. Part 2: prevention and therapy. Nat Rev Cardiol. 2011;9(2):79-90. https://doi.org/1 0.1038/nrcardio.2011.148.

5. Kipshidze N, Dangas G, Tsapenko M, Moses J, Leon MB, Kutryk M, Serruys P. Role of the endothelium in modulating neointimal formation: vasculoprotective approaches to attenuate restenosis after percutaneous coronary interventions. J Am Coll Cardiol. 2004;44(4):733-9. https://doi.org/1 0.1016/j.jacc.2004.04.048.

6. Lim WH, Seo WW, Choe W, Kang CK, Park J, Cho HJ, Kyeong S, Hur J, Yang HM, Cho HJ, Lee YS, Kim HS. Stent coated with antibody against vascular endothelial-cadherin captures endothelial progenitor cells, accelerates reendothelialization, and reduces neointimal formation. Arterioscler Thromb Vasc Biol. 2011;31(12):2798-805. https://doi.org/10.1161/ATVBAHA.111.22 6134.

7. Padfield GJ, Newby DE, Mills NL. Understanding the role of endothelial progenitor cells in percutaneous coronary intervention. J Am Coll Cardiol. 2010;55(15):1553-65. https://doi.org/10.1016/j.jacc.2009.10.070.

8. Simard T, Jung RG, Motazedian P, Di Santo P, Ramirez FD, Russo JJ, et al. Progenitor cells for arterial repair: incremental advancements towards therapeutic reality. Stem Cells Int. 2017;2017:8270498.

9. Lan H, Wang Y, Yin T, Wang Y, Liu W, Zhang X, Yu Q, Wang Z, Wang G. Progress and prospects of endothelial progenitor cell therapy in coronary stent implantation. J Biomed Mater Res B Appl Biomater. 2016;104(6):123747. https://doi.org/10.1002/jbm.b.33398.

10. Arcangeli A, Lastraioli E, Piccini B, D'Amico M, Lenzi L, Pillozzi S, et al. Circulating endothelial progenitor cells in type 1 diabetic patients: relation with patients' age and disease duration. Front Endocrinol. 2017;8:278. https://doi.org/10.3389/fendo.2017.00278.

11. Wils J, Favre J, Bellien J. Modulating putative endothelial progenitor cells for the treatment of endothelial dysfunction and cardiovascular complications in diabetes. Pharmacol Ther. 2017;170:98-115. https://doi.org/10.1016/j.pha rmthera.2016.10.014.

12. Gallagher KA, Liu ZJ, Xiao M, Chen H, Goldstein LJ, Buerk DG, Nedeau A, Thom $\mathrm{SR}$, Velazquez OC. Diabetic impairments in NO-mediated endothelial 
progenitor cell mobilization and homing are reversed by hyperoxia and SDF-1 alpha. J Clin Invest. 2007;117(5):1249-59. https://doi.org/10.1172/JCl29710.

13. Sorrentino Sajoscha A, Bahlmann Ferdinand H, Christian B, et al. Oxidant stress impairs in vivo reendothelialization capacity of endothelial progenitor cells from patients with type 2 diabetes mellitus: restoration by the peroxisome proliferator-activated receptor-gamma agonist rosiglitazone. Circulation. 2007;116:163-73.

14. Masaaki I, Hideya T, Jun A, et al. Endothelial progenitor thrombospondin-1 mediates diabetes-induced delay in reendothelialization following arterial injury. Circ Res. 2006;98:697-704.

15. Yan X, Cai S, Xiong X, Sun W, Dai X, Chen S, Ye Q, Song Z, Jiang Q, Xu Z. Chemokine receptor CXCR7 mediates human endothelial progenitor cells survival, angiogenesis, but not proliferation. J Cell Biochem. 2012;113(4): 1437-46. https://doi.org/10.1002/jcb.24015. PMID: 22173725

16. Balabanian K, Lagane B, Infantino S, Chow KY, Harriague J, Moepps B, et al, The chemokine SDF-1/CXCL12 binds to and signals through the orphan receptor RDC1 in T lymphocytes. J Biol Chem. 2005;280(42):35760-6. https:// doi.org/10.1074/jbc.M508234200.

17. Dai X, Tan Y, Cai S, Xiong X, Wang L, Ye Q, Yan X, Ma K, Cai L. The role of CXCR7 on the adhesion, proliferation and angiogenesis of endothelial progenitor cells. J Cell Mol Med. 2011;15(6):1299-309. https://doi.org/1 0.1111/j.1582-4934.2011.01301.x.

18. Dai $X$, Yan X, Zeng J, Chen J, Wang Y, Chen J, Li Y, Barati MT, Wintergerst KA, Pan K, Nystoriak MA, Conklin DJ, Rokosh G, Epstein PN, Li X, Tan Y. Elevating CXCR7 improves angiogenic function of EPCS via Akt/GSK-3beta/ Fyn-mediated Nrf2 activation in diabetic limb ischemia. Circ Res. 2017; 120(5):e7-e23. https://doi.org/10.1161/CIRCRESAHA.117.310619.

19. Zhang XY, Su C, Cao Z, Xu SY, Xia WH, Xie WL, Chen L, Yu BB, Zhang B, Wang $Y$, Tao J. CXCR7 upregulation is required for early endothelial progenitor cell-mediated endothelial repair in patients with hypertension. Hypertension. 2014;63(2):383-9. https://doi.org/10.1161/HYPERTENSIONA HA.113.02273.

20. Loboda A, Damulewicz M, Pyza E, Jozkowicz A, Dulak J. Role of Nrf2/HO-1 system in development, oxidative stress response and diseases: an evolutionarily conserved mechanism. Cell Mol Life Sci. 2016;73(17):3221-47. https://doi.org/10.1007/s00018-016-2223-0.

21. Leinonen HM, Kansanen E, Polonen P, Heinaniemi M, Levonen AL. Role of the Keap1-Nrf2 pathway in cancer. Adv Cancer Res. 2014;122:281-320. https://doi.org/10.1016/B978-0-12-420117-0.00008-6.

22. Wang RY, Liu LH, Liu H, Wu KF, An J, Wang Q, Liu Y, Bai LJ, Qi BM, Qi BL, Zhang L. Nrf2 protects against diabetic dysfunction of endothelial progenitor cells via regulating cell senescence. Int J Mol Med. 2018;42(3): 1327-40. https://doi.org/10.3892/ijmm.2018.3727.

23. Li X, Chen C, Wei L, Li Q, Niu X, Xu Y, Wang Y, Zhao J. Exosomes derived from endothelial progenitor cells attenuate vascular repair and accelerate reendothelialization by enhancing endothelial function. Cytotherapy. 2016; 18(2):253-62. https://doi.org/10.1016/j.jcyt.2015.11.009.

24. Long $P$, Sun F, Ma $Y$, Huang $Y$. Inhibition of CXCR4 and CXCR7 for reduction of cell proliferation and invasion in human endometrial cancer. Tumour Biol. 2016;37(6):7473-80. https://doi.org/10.1007/s13277-015-4580-y.

25. Hirschi Karen K, Ingram David A, Yoder Mervin C. Assessing identity, phenotype, and fate of endothelial progenitor cells. Arterioscler Thromb Vasc Biol. 2008;28(9):1584-95. https://doi.org/10.1161/ATVBAHA.107.155960.

26. Jin H, Chang-Hwan Y, Hyo-Soo K, et al. Characterization of two types of endothelial progenitor cells and their different contributions to neovasculogenesis. Arterioscler Thromb Vasc Biol. 2004;24:288-93.

27. Ingram David A,Mead Laura E,Tanaka Hiromi et al. Identification of a novel hierarchy of endothelial progenitor cells using human peripheral and umbilical cord blood .Blood, 2004, 104: 2752-2760, 9, doi: https://doi.org/1 0.1182/blood-2004-04-1396.

28. Eduard S, Timothy W, Hung-Fat T, et al. New insights on endothelial progenitor cell subpopulations and their angiogenic properties. J Am Coll Cardiol. 2008:51:669-71.

29. Pei Chong Zhe, Liu Bo, Li Ye Ting et al. MicroRNA-126 protects against vascular injury by promoting homing and maintaining stemness of late outgrowth endothelial progenitor cells. Stem Cell Res Ther, 2020, 11: 28.

30. Andrzej P, Gerrit G, David S, et al. Endothelial progenitor cells--potential new avenues to improve neoangiogenesis and reendothelialization. Int Rev Cell Mol Biol. 2013;306:43-81.

31. Masayasu I, Makoto S, Toshiaki N, et al. Diverse contribution of bone marrow-derived late-outgrowth endothelial progenitor cells to vascular repair under pulmonary arterial hypertension and arterial neointimal formation. J Mol Cell Cardiol. 2015;86:121-35.

32. Zhi C, Herrmann Sandra MS, Xiangyang Z, et al. Preserved function of lateoutgrowth endothelial cells in medically treated hypertensive patients under well-controlled conditions. Hypertension. 2014;64:808-14.

33. Yoshiyasu M, Toshiaki N, Masayasu l, et al. Angiogenic potential of early and late outgrowth endothelial progenitor cells is dependent on the time of emergence. Int J Cardiol. 2015;186:305-14.

34. Han X, Tao Y, Deng Y, Yu J, Sun Y, Jiang G. Metformin accelerates wound healing in type 2 diabetic db/db mice. Mol Med Rep. 2017;16(6):8691-8. https://doi.org/10.3892/mmr.2017.7707.

35. Hu L, Dai SC, Luan X, Chen J, Cannavicci A. Dysfunction and therapeutic potential of endothelial progenitor cells in diabetes mellitus. J Clin Med Res. 2018;10(10):752-7. https://doi.org/10.14740/jocmr3581w.

36. Ayako U-K, Bryan MK, Julia W, et al. CXCR7 agonists inhibit the function of CXCL12 by down-regulation of CXCR4. Biochem Biophys Res Commun. 2013:431:772-6.

37. Angélique $L$, Karl B, Françoise $B$, et al. CXCR7 heterodimerizes with CXCR4 and regulates CXCL12-mediated G protein signaling. Blood. 2009;113:608593.

38. Décaillot Fabien M, Kazmi Manija A, Ying L, et al. CXCR7/CXCR4 heterodimer constitutively recruits beta-arrestin to enhance cell migration. J Biol Chem. 2011;286:32188-97.

39. Zabel Brian A, Yu W, Susanna L, et al. Elucidation of CXCR7-mediated signaling events and inhibition of CXCR4-mediated tumor cell transendothelial migration by CXCR7 ligands. J Immunol. 2009;183:3204-11.

40. Maciej T, Rui L, Marcin W, et al. CXCR7: a new SDF-1-binding receptor in contrast to normal CD34(+) progenitors is functional and is expressed at higher level in human malignant hematopoietic cells. Eur J Haematol. 2010; 85:472-83.

41. Nicole HT, Valentin G, Ronit P, et al. A crosstalk between intracellular CXCR7 and CXCR4 involved in rapid CXCL12-triggered integrin activation but not in chemokine-triggered motility of human T lymphocytes and CD34+ cells. J Leukoc Biol. 2008;84:1130-40.

42. Antonio S-AJ, Sammy H, Wiebke M, et al. Cxcr7 controls neuronal migration by regulating chemokine responsiveness. Neuron. 2011;69:77-90.

43. Ming-Bao S, Yu X-J, Zhu G-X, et al. Transfection of HGF gene enhances endothelial progenitor cell (EPC) function and improves EPC transplant efficiency for balloon-induced arterial injury in hypercholesterolemic rats. Vascul Pharmacol. 2009:51:205-13.

44. Hu CH, Ke X, Chen K, Yang DY, Du ZM, Wu GF. Transplantation of human umbilical cord-derived endothelial progenitor cells promotes reendothelialization of the injured carotid artery after balloon injury in New Zealand white rabbits. Chin Med J. 2013;126(8):1480-5.

\section{Publisher's Note}

Springer Nature remains neutral with regard to jurisdictional claims in published maps and institutional affiliations.

\section{Ready to submit your research? Choose BMC and benefit from:}

- fast, convenient online submission

- thorough peer review by experienced researchers in your field

- rapid publication on acceptance

- support for research data, including large and complex data types

- gold Open Access which fosters wider collaboration and increased citations

- maximum visibility for your research: over $100 \mathrm{M}$ website views per year

At $\mathrm{BMC}$, research is always in progress.

Learn more biomedcentral.com/submissions 